\title{
Ultra-rapid non-invasive clinical nano-diagnostic of inflammatory diseases
}

\author{
Mihaela Rusu ${ }^{1}$,Alexander Dulebo ${ }^{4}$,Adelina Curaj ${ }^{1,2,3}$, Elisa A. Liehn ${ }^{1 *}$ \\ ${ }^{1}$ Institute for Molecular Cardiovascular Research (IMCAR), RWTH Aachen University, Germany \\ ${ }^{2}$ Department of Thoracic, Cardiac and Vascular Surgery, RWTH Aachen University, Germany \\ ${ }^{3}$ Victor Babes National Institute of Pathology, Bucharest, Romania \\ ${ }^{4}$ Bruker Nano Surfaces Division, Karlsruhe, Germany
}

*Correspondence to: Dr. Elisa A. Liehn, Institute for Molecular Cardiovascular Research, University Hospital Aachen, Germany; Phone: +49-241-80 35983; Fax: +49-241-80 82716; Email: eliehn@ukaachen.de;

Submitted: March 2, 2014; Revised: May 21,2014; Accepted: May 27, 2014; Published: May 28, 2014;

Citation: Rusu M, Dulebo A, Curaj A, Liehn EA. Ultra-rapid non-invasive clinical nano-diagnostic of inflammatory diseases. Discoveries Reports 2014, Sep-Dec; 1(1): e2. DOI: 10.15190/drep.2014.2

\section{ABSTRACT}

Several types of pathologies induce modified inflammatory responses in the organism, accompanied by changes in the circulating immune cells, with significant late effects and impacts on tissues organs and whole organism. Despite the significant progress of various diagnostic tools addressing many pathological situations, they still require elaborated and time-consuming laboratory work and analysis protocols.

OBJECTIVES: Within the present study a new rapid atomic force microscopy (AFM) method based on concomitant isolation and fixation of specific living blood cell-type combined with direct AFM assessment is proposed. AFM provides a complex data overview on cell morphology in combination with quantification of morphometric parameters. Thus, it makes possible a fine differentiation between disease activated- and normal-living cells.

METHODS AND RESULTS: Blood monocytes are specifically isolated from the whole blood sample using CD14 antibody-coated magnetic beads, then immobilized on freshly cleaved mica surface and studied by means of environmental-ScanAsyst-AFM method. The cells were isolated, immobilized and washed in one step. Overview AFM images convey information to morphometric parameters such as surface roughness, height step, volume and aspect ratio, while higher resolution images resolve subsurface morphological features of human blood monocytes.

CONCLUSIONS: A rapid method of isolation and selective labeling the blood monocytes was developed, thus enabling a direct morphological assessment with AFM. Series of morphometric parameters, such as surface roughness, height step, volume and aspect ratio are assigned and studied as potential diagnosis parameters. Multiplatform parameters noticeably help to differentiate monocytes present in different media as well as may be important for early diagnosis and later effective treatment. Such novel and rapid nano-diagnosis tools may constitute a "turning point" in prevention and disease detection from early stages before clinical symptoms appear, conveying towards valuable and indispensable clinical tools.

Keywords: atomic force microscopy (AFM), magnetic beads, leukocyte subpopulation, nanodiagnostic

\section{INTRODUCTION}

Several types of pathologies, e.g. cancer ${ }^{1}$, cardiovascular $^{2}$, 3 , renal $^{4,} 5$, hepatic ${ }^{6,7}$, etc, are associated with modified inflammatory response in organism. The inflammatory response is based on the interaction between specific sub-populations of 
monocytes and lymphocytes ${ }^{8-10}$. Each type of disease modifies these interactions leading to certain pattern recognition for individual sub-populations, which are specifically activated and modified at the molecular level ${ }^{8-11}$. In this context, monocytes can be recognized and identified by the presence or absence of specific biomarkers, such as the cluster of differentiation (CD), well known as targets for immunophenotyping of cells. These markers are used to associate cells with certain immune functions and specific diseases.

Although such specific interactions are known for most diseases, current diagnosis methods used are laborious and expensive. Moreover, none of the clinical accessible methods gives a rapid recognition of cellular changes at the molecular level. The development of new methods to detect early molecular changes in
Atomic force microscopy as a new rapid analytical tool of molecular diagnosis with great clinical impact molecular forces at the single molecule level ${ }^{19,}{ }^{20}$. It is well known that AFM becomes more and more popular into cell biology monitoring any modifications at micro- and nano-level in cell biology ${ }^{21}$. Compared to other analytical like focusedion-beam scanning electron microscopy (FIB-eSEM) and clinical methods such as flow cytometry, AFM is an accessible tool providing multiple morphological information such as surface topography and volume of cells, surface and intracellular modifications, etc. Currently, effort is spent to support diagnosis in an early stage, by using nano-diagnosis tools, among which AFM is aimed to 22. Moreover, quantitative changes of the cell membrane, such as elasticity, permeability and antigen presentation can rigorously be determined $^{22-27}$. Together, all these data differentiate between disease activated- and normal- living cells. In different tissues without invasive intervention is therefore very important in clinical diagnosis. Some of them proved the effectiveness in detecting local molecular changes such as contrast-enhanced ultrasound or imaging molecule-associated endothelial activation. Contrast-enhanced ultrasound present the benefit of quantitatively evaluate, e.g. tumor angiogenesis-associated molecules using labeled micro-bubbles ${ }^{12}$, 13 . For example, imaging molecule-associated endothelial activation after a high shear stress ${ }^{14,} 15$ is applied, shows a high application potential towards detection of earlier atherosclerotic lesions. Other classical methods have known huge development, as positron emission tomography imaging ${ }^{16}$, computer tomography or magnetic resonance imaging ${ }^{17,18}$. For instance, most electron microscopes investigate the morphology of delicate specimens under vacuum, after staining with a heavy metal compound such as osmium tetroxide or uranyl acetate. The ability of staining reagents to stain the soft tissues is difficult to be controlled, and therefore electron microscopy studies deal primarily with sample preparation and handling, which is a key issue to recording artifact-free images. However, these are expensive and did not pass the experimental stage.

In the last 20 years, atomic force microscopy (AFM) has emerged as a powerful technique for biological research giving ultrahigh resolution structural information and allowing measurements of general, established sample preparation protocols are mandatory for AFM analysis. This includes (i) isolation of specific sub-population which usually makes use of advanced lab methods, such as flow cytometry sorting, (ii) substrate immobilization on glass or functionalized substrates. These steps are labeled intensive thereby being time consuming, and therefore rather costly.

Therefore, herein a new rapid method based on concomitant isolation and fixation of living specific blood cell-type is proposed. Since all the methods are focused on tissue investigations, many cancer diagnostics are based on the existence of specific cells in the blood stream. Our new method has a great potential to be implemented as an efficient clinical diagnostic tool with a low cost and rapid results. It involves a bare surface of freshly cleaved mica surface, which offers the instant isolation of the target cell and a concomitant immobilization thus enabling a direct AFM analysis. Since the treatment which started in early stages, before clinical symptoms appeared, improve significantly the prognostic and the development of disease, finding new rapid analytical tools of molecular diagnosis are of a great clinical importance.

\section{MATERIAL AND METHODS}

Sampling and magnetic beads positive isolation of CD-14 positive monocytes. Blood samples are collected from healthy volunteers from the fingertip, 
using a sterile needle after proper disinfection. The first blood drop is removed and the next three drops are collected and mixed with $10 \mu \mathrm{l}$ of CD14 Antibody-conjugated MicroBeads (CD14 Antibody Microbeads, human, Miltenyi Biotec, \#130-050201), and left for $5 \mathrm{~min}$ at room temperature.

Cell preparation for analysis. Aliquots of CD14marked monocytes were adsorbed directly on freshly cleaved mica surface previously attached to a metallic AFM puck. The loosely bound specimens and cell fragments were partially removed by successively washing with phosphate buffered saline (PBS, 240-320 mOsmol/kg, Dulbecco's, Germany) at $\mathrm{pH}$ 7.4. The metallic AFM puck enables a good attachment of micro-size CD14-marked monocytes such that any cell movement during tapping is prevented. All AFM measurements were conducted in an isotonic solution of phosphate buffer saline (PBS) and a hypertonic solution of natrium chloride $(\mathrm{NaCl})$ at a concentration of $3 \% \mathrm{w} / \mathrm{v}$ and 900 $\mathrm{mOsm} / \mathrm{L}$, respectively.

AFM analysis. All experiments were carried out with a Digital Instruments MultiMode microscope in conjunction with a Nanoscope $\mathrm{V}$ controller system Veeco (Bruker, Santa Barbara, CA, USA). A "J" scanner with a lateral movement of approximately $125 \mu \mathrm{m}$ was used. All measurements were conducted in aqueous salt solution, near to physiological conditions such as in isotonic PBS solution and in hypertonic $\mathrm{NaCl}$ solution at a concentration of $3 \% \mathrm{w} / \mathrm{v}$ and $900 \mathrm{mOsm} / \mathrm{L}$, using the Bruker liquid cell. All images are acquired at room temperature $\left(23^{\circ} \mathrm{C}\right)$. ScanAsyst-Fluid probes (Bruker, Camarillo, CA, USA) were used with the characteristics of operating at a nominal resonance frequency of $150 \mathrm{kHz}$, a nominal spring constant of $0.7 \mathrm{~N} / \mathrm{m}$ and a nominal tip radius of about $20 \mathrm{~nm}$. Imaging parameters including setpoint, gain and scan rate were determined by ScanAsyst and then adjusted manually to optimize image quality. Vibrational/ acoustic shielding was achieved by placing the microscope into the acoustic isolation chamber (Bruker, Santa Barbara, CA, USA) during scanning. The ScanAsyst software enables simultaneously recording the Height, PeakForce Error, InPhase and Quadrature data channels. Height map, bearing analysis and step height, were off-line analyzed after the images were subjected to a first order plane-fit by means of NanoScope ver. 7.30 software (Bruker, Santa Barbara, CA, USA). Cell volume was estimated by means of bearing analysis according to the protocol model described by Braga and Ricci ${ }^{28}$.

Ten successive scannings were performed, corresponding to ten cells, and the results are presented by mean \pm standard deviation.

\section{RESULTS}

Monocyte morphology in isotonic conditions

The CD14 labeled cellular suspension was carefully attached to the freshly cleaved mica surface and mounted to the AFM sample stage, as described in material and methods section. Individual monocyte blood cells were consistently found to adhere firmly to the underlying mica substrate and were unaffected under imaging conditions (stable to prolonged scanning). Monocytes were investigated by means of ScanAsyst mode AFM under liquid conditions (Figure 1A). The landscape of the monocyte surface revealed in the 3D-topology image shows a rough surface next to possible cell debrides. The investigated CD14 marked cell appears to have an elongated shape with a higher topology in the center developing lower to the peripheral region (Figure 1B).

InPhase image illustrates a high contrast color gradient between two darker, circular domain structures and the brighter area assigns the membrane cell structure next to portions from the cells, which represents the micro-beads onto the cell membrane (Figure 1C). The mean size of these features is approximately $250 \mathrm{~nm}$, representing aggregates of micro-beads (with a nominal size of 60 $\mathrm{nm}$, according to the manufacturer). Topological variation of the cell membrane can off-line be extracted from the height map analysis by drawing a line across the investigated features (Figure 1D). In addition, a comparison between topology and InPhase variation indicates that the beads are located fairly on top of the cell membrane rather being embedded into the membrane structure of the cell (Figure 1E), and did not change the top surface topology of cells. However, the beads positioned at the cell-substrate interface may deform the monocyte shape, presumably elongating their shape upon adsorption.

Fine structural details at the membrane aqueous liquid interface are in general more easily observed in the PeakForce error map (Figure 1F) compared to in the height image (Figure 1G). The PeakForce error map illustrates that the monocytes appear to have an irregular structure, prominently 
A

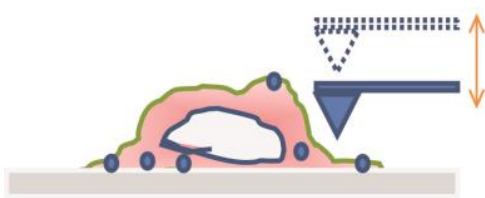

B

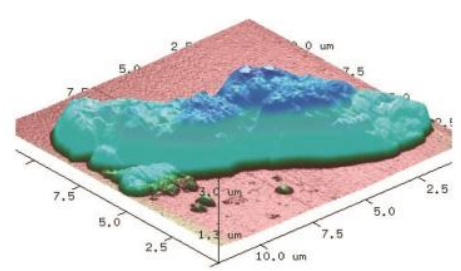

C

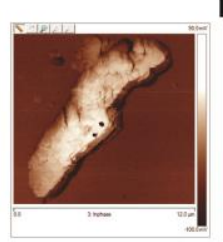

$\mathbf{F}$

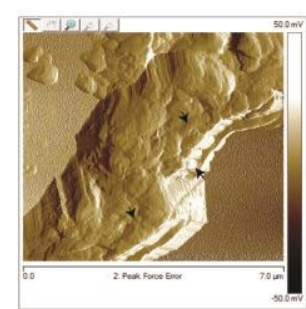

E
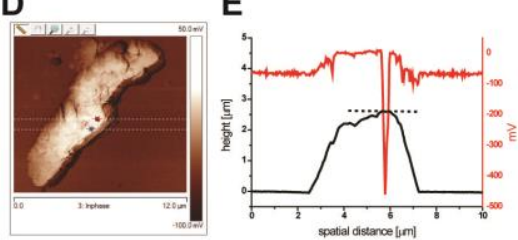

G

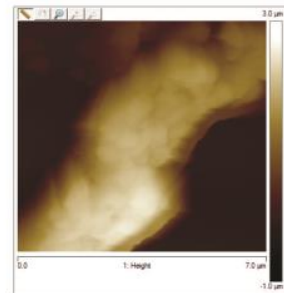

Figure 1. Scheme of the adherence of CD14-labeled living monocytes on planar surface under controlled low tapping force (A) and 3D-morphology overview of surface membrane of individual human CD14-monocyte type cell in isotonic solution (B). InPhase image of individual CD14-labelled living monocytes in isotonic solution (C) with two individual section lines across the identified beads-like structure (D). Beads location is identified by topology (black curve) and InPhase profiles (red curve) along one sectioned line (horizontal dotted line, E). PeakForce error maps (F) and morphological details (G) of the top surface living monocytes. Fine subsurface structures are marked with head black arrows (F). Different brightness relates to different heights within the cell topology whereas in InPhase channel different working amplitudes are detected corresponding to different cell surface compositions.

rough and ruffle with a typical cross-striation and a step-like morphology.

\section{Morphometric parameters of monocytes under isotonic conditions}

The envelope template of individual monocyte attached onto the planar support can be represented in different ways such as: (i) 3D-topography, a real topology visualization given by the Nanoscope software, (ii) by assembling each line profile sectioning along the monocyte until a full profile is obtained, as well as (iii) more advanced computational programs. We applied the second approach and importing each line profile as ASCII files in a successive order into Origin 7 (OriginLab, Northampton, MA, USA) (Figure 2A). The labeled - cell shapes have a mean high aspect ratio value of approximately 3 , as being determined by the ratio between major and minor axis. A systematic estimation of the mean value of the cell size is represented in histogram in Figure 2B. The mean value of elliptical type monocytes has the major axis size approximately $15 \mu \mathrm{m}$, whereas the mean value of the minor axis is approximately $5 \mu \mathrm{m}$. The cell volume can be drawn from bearing analysis. Estimated bearing volume is approximately $70 \mathrm{fl}$ correponding to a maximum bearing area of about $100 \%$ (Figure 2C).
Monocytes morphology under hyperosmotic stress conditions

Monocytes suspended in hypertonic solution displays an unusual shape (Figure 3A, Figure 3B). Salt affects not only the morphology of the cell membrane but also their surface roughness.

Though a small step height value of approximately $0.4 \mu \mathrm{m}$ is obtained in case of monocytes in hypertonic solution compared to $1.1 \mu \mathrm{m}$ in isotonic solution (Figure 3C), smaller values of cell volume are computed in case of hypertonic compared to isotonic solution (Figure 3D). The cell volume can be drawn from the bearing analysis. The bearing volume is about $47 \mathrm{fl}$ for a constant bearing area of $100 \%$. This value weights the average value over 6 image boxes (Table 1, Figure 3E).

The volume value computed with the bearing analysis tool is in line with roughness and step height parameters. Therefore, next to surface roughness and step height parameters, the bearing analysis provides relevant values of the geometrical parameters like bearing volume. A higher resolution image of the membrane cell surface shows characteristic circular-shape concave structures of approximately $300 \mathrm{~nm}$ (pink marked points, Figure 3F). Although the measurments are conducted under a minimal force (soft tapping conditions), small prolognated features appears as a consequence of 
A

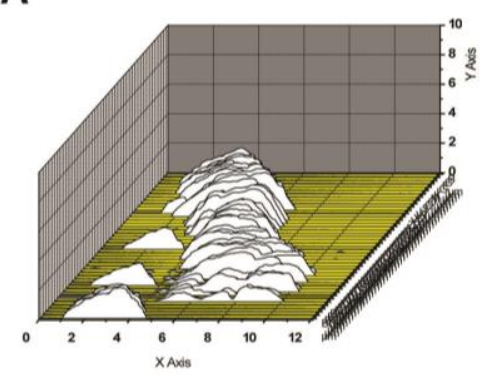

B

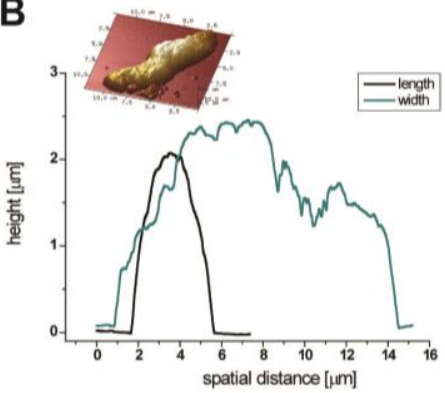

C

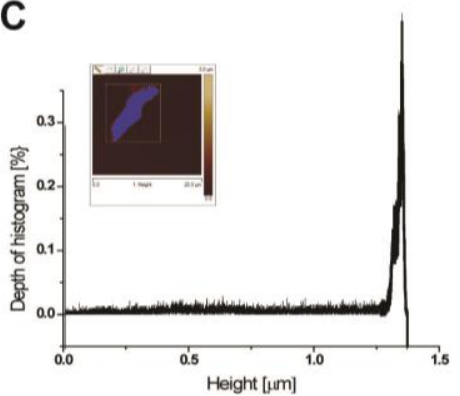

Figure 2. Topology structure computed from adjacent section lines across minor axis of healthy individual human living monocytes in isotonic solution (A); Topology spectra of healthy individual human living monocytes in isotonic solution after line section across the cell along the major (blue curve, B) and minor axis (black curve, B); Inset represents the 3D overview of the monocyte in isotonic solution (B). Depth histograms of healthy individual human living monocytes type cell calculated from their mean values $(C)$. Inset represents the processed height image of living monocyte $(C)$.

tip-cell membrane interaction as shown previously ${ }^{29}$, 30 .

\section{Roughness Analysis}

Local variations of surface topology are expressed as mean values of two roughness parameters: root mean square, $\mathrm{Rq}$, and arithmetic average of absolute height difference values, $\mathrm{Ra}$, (Eq. 1 and 2). Rq represents the height deviations from the mean image data plane expressed as:

$$
\begin{aligned}
& R q=\sqrt{\frac{\sum Z_{i}^{2}}{N}} \\
& R a=\frac{1}{N} \sum_{J=1}^{N}|Z i|
\end{aligned}
$$

where: $\mathrm{Zi}$ represents the current $\mathrm{z}$ values,

$\mathrm{N}$ number of points within the box cursor.

It is particularly observed that hypertonic solution affects the morphology of monocytes displaying a rougher surface compared to isotonic solution. The roughness values of the membrane surface at the interface of two different aqueous liquids demonstrate for such complicated biological interfaces the local rearrangement of the cell membrane when in contact with different types of liquids. This phenomenon is observed in InPhase images displayed in Figure 3A, Figure 3B. The use of $\mathrm{Ra}$ and $\mathrm{Rq}$ as a measurement of cell surface status is likely to reflect the status of the cell surface exposed to different external stimuli. The estimation of the roughness of a cell surface $(\mathrm{Rq}, \mathrm{Ra})$ provides information regarding the cell behavior to external stimuli, their dynamics in different microenvironments, cell development stage, etc. ${ }^{31}$.

The image is corrected by applying the $1^{\text {st }}$ order fitting functions after that the investigated object is excluded from fitting, followed by calculation of two surface roughness parameters, Ra and Rq. Thus, it enables a corrected determination of geometrical parameters of the cells (size, height, volume, aspect ratio, etc). A threshold height value of $150 \mathrm{~nm}$ and $100 \mathrm{~nm}$ for cells in isotonic and hypertonic solutions, respectively, provided the

Table 1: Surface roughness parameters ( \pm standard deviation) of CD14-labeled monocytes successively estimated in the presence of isotonic and hypertonic solutions.

\section{Roughness parameters \\ Monocytes in isotonic \\ Monocytes in hypertonic}

\begin{tabular}{|l|c|c|}
\hline $\mathrm{Rq}(\mathrm{nm})$ & $34 \pm 8 \%$ & $85 \pm 12 \%$ \\
\hline \hline $\mathrm{Ra}(\mathrm{nm})$ & $27 \pm 6 \%$ & $55 \pm 15 \%$ \\
\hline \hline Bearing area $\%$ & 100 & 100 \\
\hline Bearing volume $\left(\mu \mathrm{m}^{3}\right)$ & $70 \pm 15 \%$ & $47 \pm 17 \%$ \\
\hline Step height $(\mu \mathrm{m})$ & $1.1 \pm 12 \%$ & $0.4 \pm 18 \%$ \\
\hline
\end{tabular}



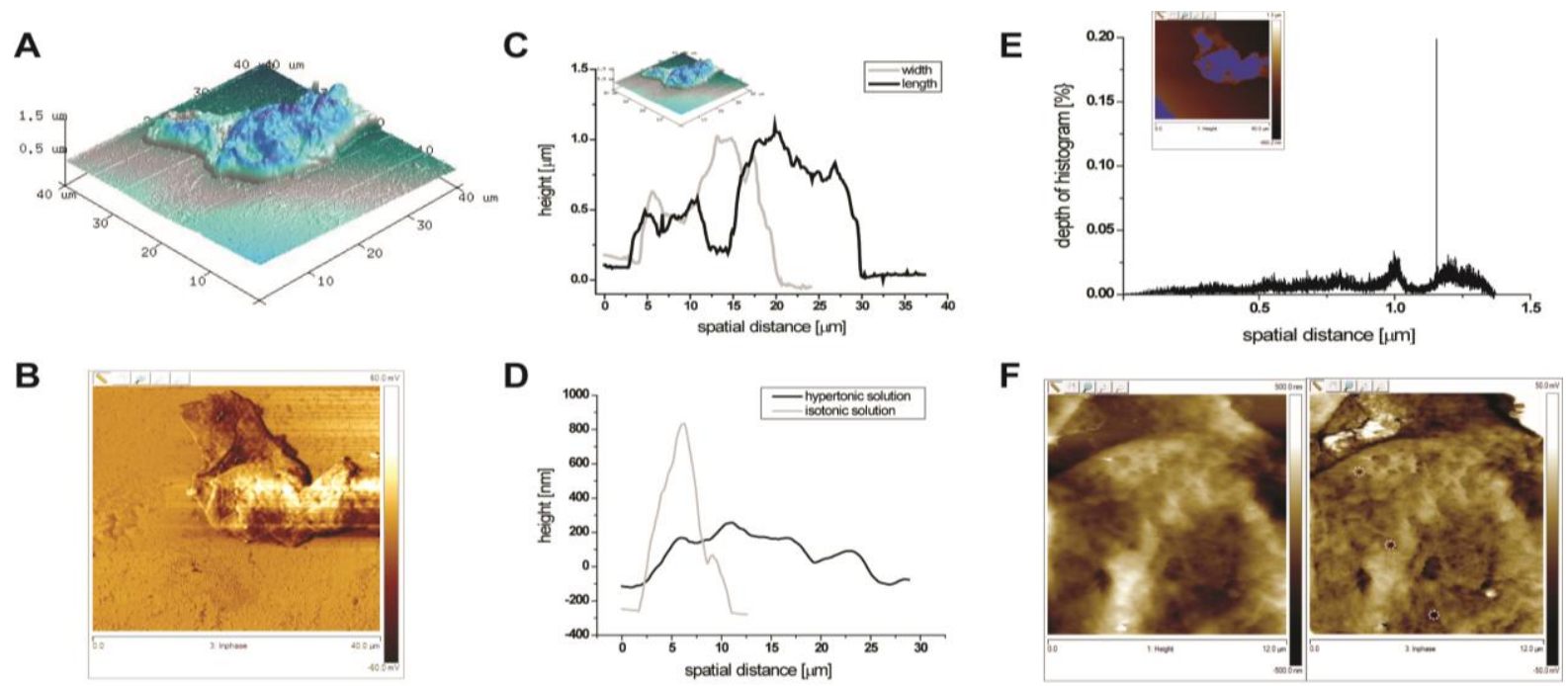

\begin{abstract}
Figure 3. 3D-morphology overview of individual healthy human CD14-monocyte in hypertonic solution (A) and the corresponding InPhase image (B); Topology spectra of healthy individual human living monocytes in hypertonic solution after line section across the cell along the major (black curve) and minor axis (gray curve, C); Topology spectra of healthy individual human living monocytes in isotonic (gray curve) and hypertonic solutions (black curve), respectively (D); Depth histograms of monocytes type cell calculated from their mean values with inset representing the processed height image of living monocyte (E); High resolution height (right) and InPhase (left) images of healthy human individual monocytes in hypertonic solution $(\mathrm{F})$; The pink circular dashed lines indicate the presence of pits-like structure with a mean size of approximately $300 \mathrm{~nm}$. Different brightness relates to different heights within the cell topology whereas in InPhase channel different tapping amplitudes are detected corresponding to different cell surface compositions.
\end{abstract}

roughness estimation of the cells. The collected mean roughness values are presented in Table 1.

\section{DISCUSSION}

In the last decades, the clinical diagnostic has made a considerable progress. New efficient devices, such as computer tomography (CT), positron emission tomography (PET) and magnetic resonance imaging (MRI), have been developed and improved, enabling a higher resolution for a better accuracy of diagnostic. Nevertheless, many pathological situations do not permit an imagistic approach diagnostic. An extended inflammatory condition, such as in chronic diseases or cancer, induces changes in circulating immune cells, with significant late effects on tissues and organs. These changes can be revealed only after elaborated and timeconsuming laboratory analysis.

In the present study, we describe an ultra-rapid non-invasive clinical nano-diagnostic method based on a detailed blood cells characterization. The method is based on the combination of a rapid blood preparation using small sample volume and a direct visualization of different levels of cell structures followed by the quantification of morphometrical parameters of blood human monocytes.
The rational of the present nano-technology method development: (i) to quantify its potential to characterizing and discriminating the blood cells and (ii) to investigate cell dynamics under external stimuli. This study may lunch further mechanism understandings and optimizations for prognosis and prevention of any blood-related disorders, particularly on diagnosis and profiling the inflammatory modifications at different degrees level. Beyond imaging, AFM provides a "real time" study of living organisms, emerging multifunctional application tools such as: morphology, molecular forces $^{19,20}$ and mechanical properties ${ }^{32,33}$.

However, AFM requires the immobilization of the cells on bare surfaces, which may influence the cell morphology ${ }^{34}$. At the moment, cultured cells on dishes, chemical fixation with glutaraldehyde/ formalin, or mechanical immobilization using gels or porous membrane require precautions when scanning to avoid cell damage or detachment during analysis $^{35,36}$.

Since sample preparation is a key issue for cell analysis, our method using antibody-conjugated magnetic beads, instantaneously isolates and immobilizes the human blood monocytes on a predetermined surface. In this way, we simplify the 
method called "Ephesia"26, providing the cells directly to the analysis. Our method also avoids a high magnetic field, which is described to change the plasma membrane structure and to reorganize the cytoskeletal components ${ }^{37}$. By isolating, immobilizing and washing the cells in one step, we significantly shorten the time for sample preparation, which does not exceed 5 minutes. Even the following measurements on AFM can require longer time and expertise at the beginning, for diagnostic purpose a fully automatic system to analyse cell ${ }^{21}$ can be combined and developed ${ }^{32,33}$.

Currently, the clinical diagnostic is based on the morphology of the cells, stained with specific antibodies, using conventional microscopic techniques. Herein, we integrate the immunohistological characterization (labelled magnetic beads) in the first step, which also represents an advantage over the other methods. Moreover, AFM can offer a high resolution, combined with precise calculation of different morphological parameters, which can not be achieved by other microscopy tools or human eye. Nevertheless, AFM can provide a large platform of information, from which only several parameters are selected to characterizing the cell, such as volume, roughness, step height and aspect ratio (Table 1). A multitude of data emphasize that these parameters are sufficient to differentiate between cells in normal and pathological states.

A current hurdle in the evaluation of cellular morphology is the lack of possibilities to import directly the property image such as geometrical parameters and surface data (roughness, height variation, etc.) into a single application. However, zrange data and section analysis tool enable the quantification of geometrical parameters such as size, shape, volume, aspect ratio, etc and to demonstrate their dependence on external stimuli. The spatial (x,y and $z$ ) co-ordinates of each pixel of $3 \mathrm{D}$-image of cells in iso- and hyper-tonic conditions allow a good estimation of the immobilized cells. Thus, the total cell volume represents the sum over all pixel-defined columns.
The shape of monocytes is usually round or oval. Due to the immobilization with magnetic beads, the shape of the labeled cell is elliptical (Figure 1). Equivalent studies, using fixation with glutaraldehide ${ }^{38}$, showed also ovoid form of the cells, however, a significant reduced volume (almost two orders of magnitude), which definitively induces changes in surface topography ${ }^{38}$. Nevertheless, differences in volume and surface microstructure of the peripheral blood monocytes are detectable after activation in pathologic conditions ${ }^{38,39}$. For example uremia induces a significant increase of monocyte volume, mean height and surface roughness compared with healthy volunteers ${ }^{38}$. Although cell volume and step height are important parameters, the surface roughness is the most relevant parameter, which is able to finely differentiate between a normal and pathological state of the cell. The roughness combined with complex cell membrane structure $^{40,} 41$ can not be analyzed with other microscopy techniques, but can precisely be quantified by means of AFM.

In the last years, many studies demonstrate the importance of the roughness in characterizing the cell morphology, corresponding to a specific function or state. The estimation of the roughness of a cell surface ( $\mathrm{Rq}, \mathrm{Ra})$ provides information regarding the cell behavior to external stimuli, their dynamics in different micro-environments, cell development stage, etc. Furthermore, surface roughness can be used to monitor the response of cells to signaling molecule. Floated cells present a higher roughness compared with adherent cells ${ }^{42}$. Similarly, T-lymphocytes in thyroid associated ophtalmopathy show a higher diameter and roughness compared with control group ${ }^{43}$. For example, drugs as paclitaxel modify the roughness of epithelia cells ${ }^{44}$, or aldosteron remodel the plasma membrane of cardiomyocytes ${ }^{45}$. Moreover, cell membrane is sensitive to changes of environmental conditions, such as salt, $\mathrm{pH}$, ionic strength, etc. Cells are shrinking or swelling, depending on suspension ion concentration, thus affecting their geometrical parameters ${ }^{46}$. In the present study, roughness in

- Concomitant immunohistological, morphological and functional analysis of blood monocytes

- Morpho-parameters describing the behavior of the living cells in normal and stress conditions

- Early stages detection of pathological changes at molecular level 
contrast to volume of cells is smaller when they are studied in isotonic compared to hypertonic solution. A decreased hydrated volume of monocytes induces a shrinkage of the interfacial membrane such that higher roughness is developed. Concomitently, the bearing analysis providing bearing volume is a relevant geometrical parameter. Therefore, we conclude that the parameters calculated in isotonic and hypertonic conditions, as a measure a cellular stress, provide valuable information about the state of the cell, differentiating between normal and pathological behaviour. In the present study, the scan speed of full frame is estimated at 7 min per frame $(20 \mu \mathrm{m} \times 20 \mu \mathrm{m})$, however, this can be reduced for future diagnosis, by optimizing working parameters.

Besides morphological features, mechanical characterization can be included as a descriptive parameter. There are many studies describing changes in stiffness of the cells in pathological conditions. For example, lymphocytes from patients with chronic lymphoblastic leukemia show lower stiffness $^{27}$, B-cell changes the mechanical responce when stimulated with antigens $\mathrm{s}^{25}$, mechanical strenght is able to regulate the T-cell ${ }^{19}$ and $\mathrm{T}$ cell responsiveness ${ }^{23}$. However, additional detailed studies are required to include machanical properties as valuable parameters in the charactherization of cell condition.

\section{Conclusions}

Our study offers a rapid tandem method based on the immunohistological, morphological and functional assessment of blood monocytes. The cell morphology is profiling on a careful selected morpho-parameters, as being assigned possible diagnosis parameters, such as surface roughness, height step, volume and aspect ratio. These parameters are describing the behavior of the living cells in normal and under stress conditions, and therefore can finely differentiate between the normal and pathological states. Such novel and rapid nanodiagnosis tools may constitute a "turning point" in prevention and disease detection from early stages until clinical symptoms appear, conveying towards valuable and indispensable clinical tool.

\section{Acknowledgments}

This study was supported by the Interdisciplinary Centre for Clinical Research IZKF Aachen (junior research group to E.A.L.) within the faculty of
Medicine at RWTH Aachen University. We thank very much to Bruker Nano Surface Division, Karlsruhe, Germany for the sustaining support information.

\section{REFERENCES}

1. De Palma M, Murdoch C, Venneri MA, et al. Tie2expressing monocytes: regulation of tumor angiogenesis and therapeutic implications. Trends in immunology. 2007;28:519-524.

2. Nahrendorf M, Swirski FK, Aikawa E, et al. The healing myocardium sequentially mobilizes two monocyte subsets with divergent and complementary functions. The Journal of experimental medicine. 2007;204:3037-3047.

3. Weber C, Zernecke A, Libby P. The multifaceted contributions of leukocyte subsets to atherosclerosis: lessons from mouse models. Nature reviews. 2008;8:802815.

4. Lin SL, Castano AP, Nowlin BT, et al. Bone marrow Ly6Chigh monocytes are selectively recruited to injured kidney and differentiate into functionally distinct populations. J Immunol. 2009;183:6733-6743.

5. Ulrich C, Heine GH, Seibert E, et al. Circulating monocyte subpopulations with high expression of angiotensin-converting enzyme predict mortality in patients with end-stage renal disease. Nephrol Dial Transplant. 2010;25:2265-2272.

6. Karlmark KR, Weiskirchen R, Zimmermann HW, et al. Hepatic recruitment of the inflammatory Gr1+ monocyte subset upon liver injury promotes hepatic fibrosis. Hepatology 2009;50:261-274.

7. Zimmermann HW, Seidler S, Nattermann J, et al. Functional contribution of elevated circulating and hepatic non-classical CD14CD16 monocytes to inflammation and human liver fibrosis. PloS one. 2010;5:e11049.

8. Liehn EA, Postea O, Curaj A, et al. Repair after myocardial infarction, between fantasy and reality: the role of chemokines. Journal of the American College of Cardiology. 2011;58:2357-2362.

9. Liehn EA, Zernecke A, Postea O, et al. Chemokines: inflammatory mediators of atherosclerosis. Archives of physiology and biochemistry. 2006;112:229-238.

10. Subimerb C, Pinlaor S, Lulitanond V, et al. Circulating CD14(+) CD16(+) monocyte levels predict tissue invasive character of cholangiocarcinoma. Clinical and experimental immunology. 2010;161:471-479.

11. Kanzler I, Liehn EA, Koenen RR, et al. Antiinflammatory therapeutic approaches to reduce acute atherosclerotic complications. Current pharmaceutical biotechnology. 2012;13:37-45.

12. Palmowski M, Huppert J, Ladewig G, et al. Molecular profiling of angiogenesis with targeted ultrasound imaging: early assessment of antiangiogenic therapy effects. Molecular cancer therapeutics. 2008;7:101-109. 
13. Palmowski M, Morgenstern $\mathrm{B}$, Hauff $\mathrm{P}$, et al. Pharmacodynamics of streptavidin-coated cyanoacrylate microbubbles designed for molecular ultrasound imaging. Investigative radiology. 2008;43:162-169.

14. Fokong S, Fragoso A, Rix A, et al. Ultrasound molecular imaging of E-selectin in tumor vessels using poly n-butyl cyanoacrylate microbubbles covalently coupled to a short targeting peptide. Investigative radiology. 2013;48:843-850.

15. Wu Z, Curaj A, Fokong S, et al. Rhodamine-Loaded Intercellular Adhesion Molecule-1-targeted Microbubbles for Dual-Modality Imaging Under Controlled Shear Stresses. Circ Cardiovasc Imaging. 2013;6:974-981.

16. Orbay H, Zhang Y, Valdovinos HF, et al. Positron emission tomography imaging of CD105 expression in a rat myocardial infarction model with (64)Cu-NOTATRC105. American journal of nuclear medicine and molecular imaging. 2013;4:1-9.

17. Buonincontri G, Methner C, Carpenter TA, et al. MRI and PET in Mouse Models of Myocardial Infarction. $J$ Vis Exp. 2013.

18. Gargiulo S, Greco A, Gramanzini M, et al. PET/CT imaging in mouse models of myocardial ischemia. Journal of biomedicine \& biotechnology. 2012;2012:541872.

19. Lim TS, Ricciardi-Castagnoli P. Single-cell force spectroscopy: mechanical insights into the functional impacts of interactions between antigen-presenting cells and T cells. Immunologic research. 2012;53:108-114.

20. Starodubtseva MN, Yegorenkov NI, Nikitina IA. Thermo-mechanical properties of the cell surface assessed by atomic force microscopy. Micron. 2012;43:1232-1238. 21. Wang Z, Liu L, Wang Y, et al. A fully automated system for measuring cellular mechanical properties. Journal of laboratory automation. 2012;17:443-448.

22. Reichlin $\mathrm{T}$, Wild $\mathrm{A}$, Durrenberger $\mathrm{M}$, et al. Investigating native coronary artery endothelium in situ and in cell culture by scanning force microscopy. Journal of structural biology. 2005;152:52-63.

23. Lim TS, Mortellaro A, Lim CT, et al. Mechanical interactions between dendritic cells and $\mathrm{T}$ cells correlate with T cell responsiveness. J Immunol. 2011;187:258265.

24. Marsh G, Waugh RE. Quantifying the mechanical properties of the endothelial glycocalyx with atomic force microscopy. J Vis Exp. 2013:e50163.

25. Natkanski E, Lee WY, Mistry B, et al. B cells use mechanical energy to discriminate antigen affinities. Science 2013;340:1587-1590.

26. Saliba AE, Saias L, Psychari E, et al. Microfluidic sorting and multimodal typing of cancer cells in selfassembled magnetic arrays. Proceedings of the National Academy of Sciences of the United States of America. 2010;107:14524-14529.

27. Skorkina MY, Fedorova MZ, Muravyov AV, et al. The use of nanomechanic sensor for studies of morphofunctional properties of lymphocytes from healthy donors and patients with chronic lymphoblastic leukemia. Bulletin of experimental biology and medicine. 2012;154:163-166.

28. Braga PC, Ricci D. Atomic Force Microscopy. Biomedical Methods and Applications. Totowa, New Jersey: Humana Press; 2004: 394.

29. Dulebo A, Preiner J, Kienberger F, et al. Second harmonic atomic force microscopy imaging of live and fixed mammalian cells. Ultramicroscopy. 2009;109:10561060 .

30. Espenel C, Giocondi MC, Seantier B, et al. Temperature-dependent imaging of living cells by AFM. Ultramicroscopy. 2008;108:1174-1180.

31. Pandiyaraj KN, Deshmukh RR, Mahendiran R, et al. Influence of operating parameters on surface properties of RF glow discharge oxygen plasma treated TiO2/PET film for biomedical application. Materials science \& engineering. 2013;36:309-319.

32. Alsteens D, Dupres V, Mc Evoy K, et al. Structure, cell wall elasticity and polysaccharide properties of living yeast cells, as probed by AFM. Nanotechnology. 2008; 19:384005.

33. Fuhrmann A, Staunton JR, Nandakumar V, et al. AFM stiffness nanotomography of normal, metaplastic and dysplastic human esophageal cells. Physical biology. 2011;8:015007.

34. Kong F, Li Z, Parks WM, et al. Cyclic mechanical reinforcement of integrin-ligand interactions. Molecular cell. 2013;49:1060-1068.

35. El Kirat K, Burton I, Dupres V, et al. Sample preparation procedures for biological atomic force microscopy. Journal of microscopy. 2005;218:199-207.

36. Francis LW, Gonzalez D, Ryder T, et al. Optimized sample preparation for high-resolution AFM characterization of fixed human cells. Journal of microscopy. 2010;240:111-121.

37. Fojt L, Klapetek P, Strasak L, et al. $50 \mathrm{~Hz}$ magnetic field effect on the morphology of bacteria. Micron. 2009;40:918-922.

38. Chen D, Gan H, Huang X, et al. Effects of peripheral blood mononuclear cells morphology on vascular calcification in uremic patients on maintenance hemodialysis. Ther Apher Dial. 2012;16:173-180.

39. Tsirpanlis G, Chatzipanagiotou S, Ioannidis A, et al. Serum and peripheral blood mononuclear cells infectious burden: correlation to inflammation and atherosclerosis in haemodialysis patients. Nephrology 2005;10:256-263.

40. Leabu M. The still valid fluid mosaic model for molecular organization of biomembranes: accumulating data confirm it. Discoveries. 2013;1:e7.

41. Nicolson GL. Update of the 1972 Singer-Nicolson Fluid-Mosaic Model of Membrane Structure. Discoveries. 2013;1:e3.

42. Zhang W, Gao Z, Shao D, et al. Atomic force microscopy analysis of progenitor corneal epithelial cells fractionated by a rapid centrifugation isolation technique. PloS one. 2013;8:e59282. 
43. Song J, Xiao LH, Hei Y, et al. Study on peripheral blood T lymphocyte cell membrane in thyroid associated ophthalmopathy by using atomic force microscopy. Chinese journal of ophthalmology. 2009;45:522-527.

44. Kim KS, Cho CH, Park EK, et al. AFM-detected apoptotic changes in morphology and biophysical property caused by paclitaxel in Ishikawa and HeLa cells. PloS one. 2012;7:e30066.

45. Kliche K, Kuhn M, Hillebrand U, et al. Direct aldosterone action on mouse cardiomyocytes detected with atomic force microscopy. Cell Physiol Biochem. 2006;18:265-274.
46. Oberleithner H, Peters W, Kusche-Vihrog K, et al. Salt overload damages the glycocalyx sodium barrier of vascular endothelium. Pflugers Arch. 2011;462:519-528.

DISCOVERIES REPORTS is a peer-reviewed, open access, online, multidisciplinary and integrative journal, publishing high impact and innovative manuscripts from all areas related to MEDICINE, BIOLOGY and CHEMISTRY;

(C) 2014, Applied Systems 